\title{
A NOTE ON A CHARACTERIZATION THEOREM FOR A CERTAIN CLASS OF DOMAINS
}

\author{
SHAFIQ UR REHMAN
}

Received 12 October, 2016

\begin{abstract}
We have introduced and studied in [2] the class of Globalized multiplicatively pinchedDedekind domains (GMPD domains). This class of domains could be characterized by a certain factorization property of the non-invertible ideals, (see [2, Theorem 4]). In this note a simplification of the characterization theorem [2, Theorem 4] is provided in more general form.
\end{abstract}

2010 Mathematics Subject Classification: 13A15; 13F05

Keywords: invertible ideal, Prüfer domain, h-local domain

Let $D$ be an integral domain. By an MNI ideal of $D$ we mean an ideal of $D$ which is maximal among the nonzero noninvertible ideals of $D$. By [6, Exercise 36, page 44], every MNI ideal is a prime ideal. Moreover, using standard Zorn's Lemma arguments, one can show that every nonzero non-invertible ideal is contained in some MNI ideal. $D$ is said to be $h$-local provided every nonzero ideal of $D$ is contained in at most finitely many maximal ideals of $D$ and each nonzero prime ideal of $D$ is contained in a unique maximal ideal of $D . D$ is called a pseudo-valuation domain $(P V D)$ if $D$ is quasi-local with maximal ideal $M$ and $M: M$ is a valuation domain with maximal ideal $M$, cf. [5] and [1, Proposition 2.5]. A two-generated domain is a domain whose ideals are two-generated. Let $D$ be a quasi-local domain with maximal ideal $M$. By [5, Theorems 2.7 and 3.5], $D$ is a two-generated PVD if and only if $D$ is a field, a DVR, or a Noetherian domain such that its integral closure $D^{\prime}$ is a DVR with maximal ideal $M$ and $D^{\prime} / M$ is a quadratic field extension of $D / M$.

In [2], we introduced and study the class of Globalized multiplicatively pinchedDedekind domains (GMPD domains). A domain $D$ is called a globalized multiplicatively pinched-Dedekind domain (GMPD domain) if $D$ is h-local and for each maximal ideal $M, D_{M}$ is a two-generated PVD, or a valuation domain with value group $\mathbb{Z} \times \mathbb{Z}$ or $\mathbb{R}$, cf. [2, Definition 2]. A Dedekind domain is a GMPD domain and the integrally closed Noetherian GMPD domain are exactly the Dedekind domains. This class of domains could be characterized by a certain factorization property of the non-invertible ideals. An h-local domain $D$ is a GMPD domain if and only if 
every two MNI ideals are comaximal and every nonzero non-invertible ideal $I$ of $D$ can be written as $I=J P_{1} \cdots P_{k}$ for some invertible ideal $J$ and distinct MNI ideals $P_{1}, \ldots, P_{k}$ uniquely determined by $I$ ([2, Theorem 4 , Remark 5]). In this note a more general simplification of this characterization theorem in provided (Theorem 2).

Throughout this note all rings are (commutative unitary) integral domains. For a domain $D, D^{\prime}$ (resp. $\bar{D}$ ) denotes the integral closure (resp. complete integral closure) of $D$. Any unexplained material is standard like in [4] or [6].

Lemma 1. Every two distinct MNI ideals of a domain D are comaximal.

Proof. Deny. Let $P_{1} \neq P_{2}$ be the MNI ideals of $D$ both contained in the maximal ideal $M$. Then $M$ is invertible and so $P_{i} \subsetneq M$ implies that $P_{i} \subsetneq \cap_{n \geq 1} M^{n}=Q$. The ideal $Q$ is invertible and prime. Indeed, if $a b \in Q$ with both $a, b \notin Q$, then there exist integers $k, l$ and the ideals $U, V$ such that $(a)=M^{k} U$ with $U \nsubseteq M$ and (b) $=M^{l} V$ with $V \nsubseteq M$. Since $a b \in M^{k+l+1}$, so $(a b)=M^{k+l+1} N$ for some ideal $N$. Combining, we get that $M^{k+l} U V=M^{k+l+1} N$. This implies that $U V=M N$ which is not possible because $U, V$ are not contained in $M$. Hence $Q$ is prime. As $P_{i} \subsetneq Q$, so $Q$ is invertible. Since any two invertible prime ideals are not comparable, so $Q=M$. This implies that $M=M^{2}$ and hence $M=D$, a contradiction.

Recall [3, Section 5.1] that a domain $D$ has pseudo-Dedekind factorization if for each nonzero non-invertible ideal $I$, there is an invertible ideal $B$ (which might be $D$ ) and finitely many pairwise comaximal primes $P_{1}, P_{2}, \ldots, P_{n}$ such that $I=$ $B P_{1} P_{2} \cdots P_{n}$ (the requirement that $n>0$ comes for free).

Theorem 1. Let $D$ be a domain such that every nonzero non-invertible ideal I of $D$ can be written as $I=J P_{1} \cdots P_{k}$ for some invertible ideal $J$ and distinct $M N I$ ideals $P_{1}, \ldots, P_{k}$. Then $D$ is h-local.

Proof. By [6, Exercise 36, page 44], every MNI ideal is a prime ideal and by Lemma $1, P_{1}, \ldots, P_{k}$ are pairwise comaximal. Hence $D$ has pseudo-Dedekind factorization, cf. [3, Section 5.1]. Now Apply [3, Corollary 5.2.14].

Theorem 2. A domain $D$ is a GMPD domain if and only if every nonzero noninvertible ideal $I$ of $D$ can be written as $I=J P_{1} \cdots P_{k}$ for some invertible ideal $J$ and distinct MNI ideals $P_{1}, \ldots, P_{k}$.

Proof. Apply Theorem 1 and [2, Theorem 4].

\section{ACKNOWLEDGEMENT}

This research is supported by the Higher Education Commission of Pakistan. 


\section{REFERENCES}

[1] D. F. Anderson and D. E. Dobbs, "Pairs of rings with the same prime ideals." Canad. J. Math., vol. 32, pp. 362-384, 1980, doi: 10.4153/CJM-1980-029-2.

[2] T. Dumitrescu and S. U. Rahman, "A class of pinched domains II," Comm. Alg., vol. 39, pp. 1394 1403, 2011, doi: 10.1080/00927871003705591.

[3] M. Fontana, E. Houston, and T. Lucas, Factoring Ideals in Integarl domains. Lecture Notes of the Unione Mathematica Italiana, Vol. 14, Springer-Verlag Berlin Heidelberg, 2013. doi: 10.1007/9783-642-31712-5.

[4] R. Gilmer, Multiplicative Ideal Theory. New York: Marcel Dekker, 1972.

[5] J. R. Hedstrom and E. G. Houston, "Pseudo-valuation domains," Pacific J. Math., vol. 75, pp. 137-147, 1978, doi: 10.2140/pjm.1978.75.137.

[6] I. Kaplansky, Commutative Rings. Chicago and London: The University of Chicago Press, 1974.

Author's address

Shafiq Ur Rehman

COMSATS Institute of Information Technology, Attock Campus, Pakistan

Current address: ASSMS, GC University Lahore, Pakistan

E-mail address: shafiq_ur_rahman2@yahoo.com, shafiq@ciit-attock.edu.pk 